\title{
Long-Reach Optical Access Technologies
}

\section{Darren P. Shea and John E. Mitchell, University College London}

\begin{abstract}
The search for a cost-effective optical access solution has yielded a number of possible solutions. This article seeks to summarize the economic arguments behind long-reach access and examines a number of leading long-reach optical access technologies.
\end{abstract}

A

s broadband uptake increases globally, the services offered are becoming increasingly bandwidth-intensive. A service that continues to increase bandwidth requirements is high-definition television (HDTV), which requires in the region of 8 $\mathrm{Mb} / \mathrm{s}$ per channel. For telecommunications (telecoms) networks to support a single channel of HDTV plus data and telephony services simultaneously, the minimum bandwidth required is approximately $10 \mathrm{Mb} / \mathrm{s}$. Currently, telecoms operators face a significant problem in that the cost of the capital expenditure to deploy traditional networks to support next generation, bandwidth-intensive services is higher than the revenues that these services generate. A passive optical network (PON) offers a different network architecture that enables the essentially unlimited bandwidth of fiber to the home (FTTH) to be utilized. PONs use a point-to-multipoint architecture to reduce cost by sharing a significant portion of the network among all customers rather than each customer having a dedicated connection as in the current point-to-point architecture. However, the cost reduction offered by a PON is not enough due to the expensive optical equipment that must be installed at each customer premises. Studies carried out in the 1990s estimated that the cost of installing fiber throughout the United Kingdom (UK) would be in the region of $£ 15$ billion [1]; a massive investment, especially for a private company [2]. FTTH was deferred in favor of incremental technologies based on DSL, which provided small increases in bandwidth without the requirement for large capital investment. These technologies reuse the copper infrastructure and use modern encoding and compression techniques, such as those developed by the Moving Pictures Experts Group (MPEG) to provide the required performance and quality of service.

\section{Long-Reach Technologies}

An alternative architecture was proposed as a more costeffective solution for an optical access network; the long-reach optical access network. The strength of optical technology is its ability to displace electronics and simplify the network by combining network tiers. The access and metro networks can be combined into one through the use of an extended backhaul fiber, possibly $100 \mathrm{~km}$ in length to incorporate protection paths and mechanisms, used with a PON. A general PON architecture consists of a shared fiber that originates from a local exchange. At a point close to the customer premises, a passive optical splitter is used to connect each customer to the main fiber. Current standardized PONs are a maximum of 20 $\mathrm{km}$ in length with 16,32, or 64-way splits for Ethernet-PON (EPON), broadband-PON (BPON), or Gigabit-PON (GPON), respectively. PON architectures with large split sizes are intended to maximize the number of components shared between all customers. Even though optical amplifiers must be used to increase the power budget, the distribution section closest to the customer remains passive. Cost savings are introduced as the synchronous digital hierarchy (SDH) rings are replaced with a single backhaul fiber. The combined access and backhaul network terminates at a core node, possibly resulting in the removal of the local exchange site. As shown in Fig. 1, the result is a highly simplified network. It is envisioned that only 100 nodes would be required for the entire UK, as opposed to the current 400 nodes [1].

A number of long-reach optical access network architectures have been developed. Initially, the networks were single channel, where a single wavelength is shared between all users, using time division multiplexing (TDM). These networks were followed by wavelength division multiplexing systems that shared a number of wavelengths between groups of users. More recently, GPON extension systems have been developed that enable a number of existing GPONs to be grouped and converted into long-reach systems with dense wavelength division multiplexing (DWDM) backhaul systems.

\section{PLANET SuperPON}

The ACTS (advanced communications and technologies and services) project, photonic local access NETwork (PLANET), was initiated in the mid 1990s. The project consisted of a consortium of telecoms operators and suppliers working in parallel to develop a cost-effective, full service access network. Entitled super passive optical network (SuperPON), the architecture - developed by investigating possible upgrades to the G.983 broadband PON (BPON) architecture - includes a large splitting factor and long-reach [3]. Findings of an economic study carried out as part of PLANET, revealed that FTTH was a cheaper option than fiber to the cabinet (FTTC) [3]. This seems counter intuitive as FTTC requires less fiber to be installed but involves extra cost for the housing of the optical network unit (ONU) at a street location rather than the customer premises. Providing FTTH through SuperPON was more cost-effective than BPON as customers share a large part of the network. Operation and maintenance costs are 70 percent lower for FTTH compared with FTTC [3]. Ser- 


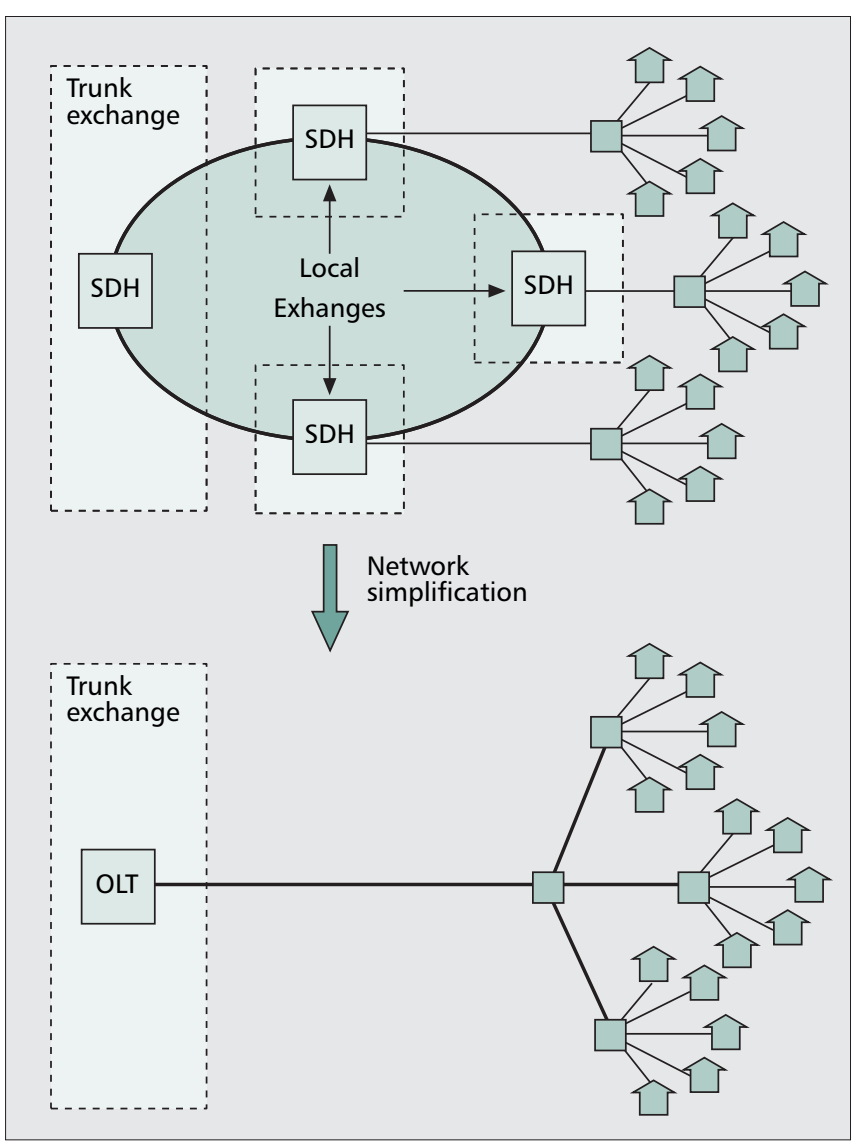

Figure 1. Network simplification, removal of SDH equipment in the metro network, using a PON with extended backhaul (OLT: optical line terminal).

vice and capacity requirements were studied, including a geographical evaluation that resulted in the split size being set at 2048 and the range set at $100 \mathrm{~km}$ for SuperPON [3].

Increasing the BPON split size from 32 to 1024 and range from $20 \mathrm{~km}$ to $100 \mathrm{~km}$ introduces large amounts of attenuation. System performance was compromised as the received signal power was reduced. To combat the performance loss, SuperPON introduced optical amplifiers into both the upstream and downstream channels. Downstream, transmission was simplified through the distribution of costs. Because the optical line terminal (OLT) was shared between all users, it was not a cost-sensitive component; therefore an expensive high power transmitter can be used. This was advantageous as a strong signal was launched into the backhaul fiber that was easily amplified by the optical amplifiers in the system ensuring a strong signal was present at the ONU receiver.

Upstream transmission was not as straight forward. Optical amplifiers provide the gain required for transmission across SuperPON architecture to be feasible. However, optical amplifiers also have a detrimental effect on performance due to unwanted optical noise, known as amplified spontaneous emission (ASE), a side effect of the amplification mechanism. The system performance can be measured in terms of the signal-to-noise ratio (SNR) which is a direct comparison of the signal power compared with the noise power. An SNR > 18.6 $\mathrm{dB}$ was required to achieve the PLANET target performance bit error rate (BER) of $10^{-9}$, using ON-OFF keying and including a $3 \mathrm{~dB}$ optical power margin [3]. The SNR at the input to the optical amplifier was a function of the split size that accounts for the majority of the distribution section loss. The ASE produced by the erbium doped fiber amplifier (EDFA) remains constant; hence the SNR was reduced as the signal was attenuated by the increased loss due to the increased split size. The maximum split size where an SNR of $18.6 \mathrm{~dB}$ can be achieved was 1024, half the original SuperPON target. The method applied to achieve a split size of 2048 was to create an amplified splitter. Instead of placing amplifiers after the splitters, optical amplifiers were included in parallel between split stages. This reduces the split loss before the optical amplifier, enabling the SNR of $18.6 \mathrm{~dB}$ to be achieved with a larger split size. However, when several optical amplifiers are placed in parallel, the effect of ASE becomes more severe. Each optical amplifier produces ASE that then is combined with the ASE contributions from the other amplifiers at the splitter, which acts as a combiner in the upstream direction [3]. This effect is known as noise funneling and increases the amount of ASE present at the receiver. The designers of SuperPON were forced to implement a complex gating protocol that reduced noise funneling by only switching on an optical amplifier when it was required to amplify a signal. Using this method, SuperPON achieved the target of a 2048-way split.

Ensuring that each of the 2048 customers was able to receive adequate bandwidth required the PLANET consortium to increase the downstream bandwidth to $2.5 \mathrm{~Gb} / \mathrm{s}$ as opposed to $622 \mathrm{Mb} / \mathrm{s}$ in BPON [3]. Upstream the capacity was increased to $311 \mathrm{Mb} / \mathrm{s}$ against $155 \mathrm{Mb} / \mathrm{s}$ in BPON. ${ }^{1} \mathrm{As}$ in BPON, downstream transmission was broadcast via a TDM protocol, and upstream capacity was shared between customers through time division multiplexing access (TDMA). PLANET opted for the TDM/TDMA protocol combination to allow wavelength division multiplexing (WDM) and wavelength division multiple access (WDMA) to be introduced at a later date, as an overlay when the demand for network capacity increases [3].

\section{Long-Reach PON}

The long-reach PON was a 1024-way split, $100 \mathrm{~km}$ reach 10 $\mathrm{Gb} / \mathrm{s}$, optical access network solution. It was developed to provide a solution to the situation forecast by British Telecom's (BT) bandwidth modeling [1]. It was a simple solution that draws on modern optical technologies. The optical split size was 1024 , which is half of that of SuperPON, but it only requires six optical amplifiers for both upstream and downstream operation as opposed to 39 required by SuperPON. The long-reach PON was completely passive in the access network section between the customer premises and the local exchange site.

An intermediate amplification site was positioned at the local exchange site immediately after the 1024-way split in the distribution section. The local exchange site was an ideal location for the intermediate amplification stage as the site already contains electrical power, and hence no electrical power must be installed in the distribution section. Because the optical amplification was performed after the split, no optical amplifiers are in parallel. Hence, noise funneling was not an issue, and therefore no complex optical gating system was required as in SuperPON [4].

Similar to the SuperPON, the distribution of cost means that transmission in the upstream is more difficult than the downstream. The distribution section has a large loss of 40.3 $\mathrm{dB}$ that results in a very low signal power at the local exchange site. To counter the attenuation of the distribution section, a dual stage intermediate amplification stage was used. The first stage consists of a low noise pre-amplifier. As the signal power was so low, the SNR is determined by the noise pro-

${ }^{1}$ Note: the BPON upstream bandwidth was later increased to $622 \mathrm{Mb} / \mathrm{s}$. 


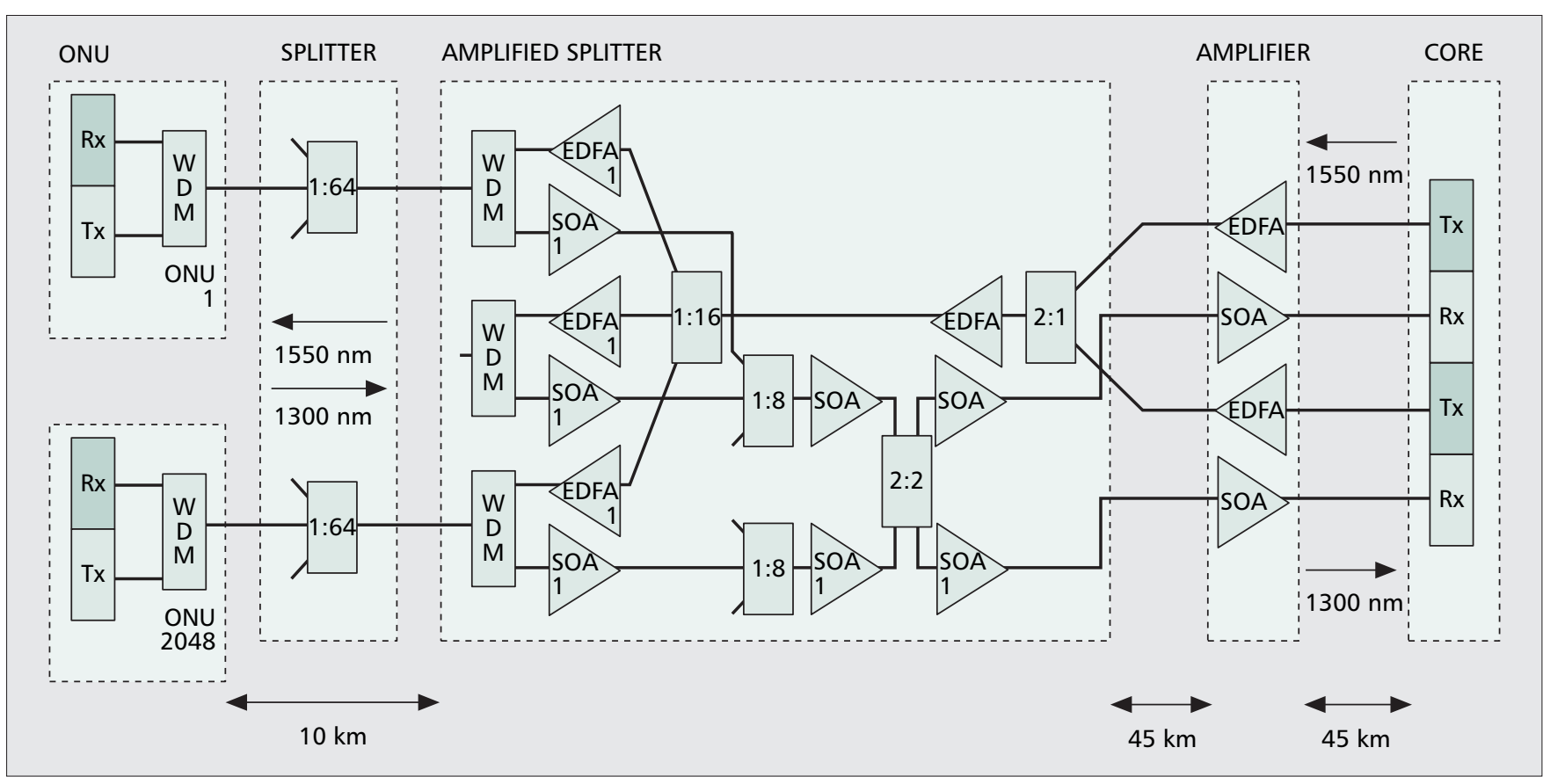

Figure 2. General SuperPON architecture [3].

duced by the optical amplifier, and hence the SNR can be maximized by minimizing the amplifier ASE.

The second amplification stage provides the signal with enough power to overcome the attenuation of the backhaul section. As the backhaul loss was $37 \mathrm{~dB}$, a relatively weak signal was present at the receiver. Hence, an optical pre-amplifier was required to increase the receiver sensitivity. The total gain supplied by the optical amplifiers was not sufficient to achieve the target performance of BER $=10^{-10}$ because of the detrimental effect of the ASE.

Forward error correction (FEC) is a technique whereby transmission errors can be detected and corrected by encoding the data and including a number of parity bits. To achieve a post-FEC performance of $\mathrm{BER}=10^{-10}$, the system requires only a pre-FEC BER of $2.9 \times 10^{-4}$. The pre-FEC error rate can be achieved by including a $2 \mathrm{~nm}$ optical filter prior to the receiver. An optical filter limits the optical frequencies that the receiver can see. Therefore, an optical filter reduces the amount of ASE present at the receiver, which increases the SNR, and therefore the performance of the system. However, the downside to using an optical filter is that the wavelength of the transmitter must be strictly defined to ensure that it can pass through the pass bandwidth of the optical filter. Unfortunately, the wavelength of a laser is temperature dependant, and therefore a temperature controller must be included to ensure the wavelength does not drift out of the filter pass band. This is not desirable as it adds additional cost to the ONU.

A cost-effective solution was found that involved using a $10 \mathrm{G}$ Ethernet transmitter. These transmitters include a thermo electric cooler (TEC) but are cost-effective due to volume production for $10 \mathrm{~Gb} / \mathrm{s}$ Ethernet applications. The downside to this transmitter was that it was designed for short-reach application of less than $40 \mathrm{~km}$. Transmission across $100 \mathrm{~km}$ at $10 \mathrm{~Gb} / \mathrm{s}$ incurred significant penalties due to chromatic dispersion in single mode fiber. A number of techniques, including dispersion compensating fiber (DCF), exist to counter dispersion penalties. The technique chosen for long-reach PON was electronic dispersion compensation (EDC), which involves placing a finite impulse response (FIR) filter after the optical receiver that filters the electrical signal in such a way as to counter the effect of the optical channel. EDC is dynamically tunable, which enables the device to compensate for each of the ONUs that are positioned at different distances from the OLT without infrastructure changes that would be required when using DCF.

Therefore, with the use of appropriate optical technologies, it was possible to achieve $10 \mathrm{~Gb} / \mathrm{s}$ transmission in the downstream and upstream channels across $100 \mathrm{~km}$ to $1024 \mathrm{cus}-$ tomers using a low cost optical transceiver in the ONU situated in the customer premises.

\section{Hybrid DWDM-TDM Long-Reach PON}

The hybrid DWDM-TDM long-reach PON, developed by Townsend \& Talli, [5] was a novel architecture that introduced DWDM into a long-reach PON architecture. The architecture enables a number of large-split, long-reach PONs, each working at different wavelengths to share the same fiber infrastructure [5]. This design combines the extended reach of optically amplified PONs with the increased number of users enabled with DWDM. DWDM relies on narrow optical filtering $(100 \mathrm{GHz}$ or $50 \mathrm{GHz})$ to separate each of the individual channels. Therefore, a suitable transmitter technology must be selected that ensures strict wavelength definition. In this application, the transmitter used an optical electro-absorption modulator (EAM) integrated with two semiconductor optical amplifiers (SOA). The optical carrier was generated at a central location in the network, enabling the network operator to have complete control over the optical wavelength of the transmitter. The first SOA was used to amplify the optical carrier at the input to the ONU, and the second was used to amplify the signal after modulation by the EAM [5].

The network infrastructure is shown in Fig. 4. The total ONU to OLT network length was $100 \mathrm{~km}$ and was divided into three sections. From the ONU to the street cabinet was the feeder fiber section that was six $\mathrm{km}$ in length. A further six $\mathrm{km}$ was situated between the street cabinet and the local exchange with an $88 \mathrm{~km}$ back haul fiber taking the total length to $100 \mathrm{~km}$. In the distribution section between the ONU and the local exchange, a number of PONs are present. Each PON was assigned a unique upstream and a unique downstream wavelength that was used between the OLT and the ONU. All of the separate PONs were then combined at the local 


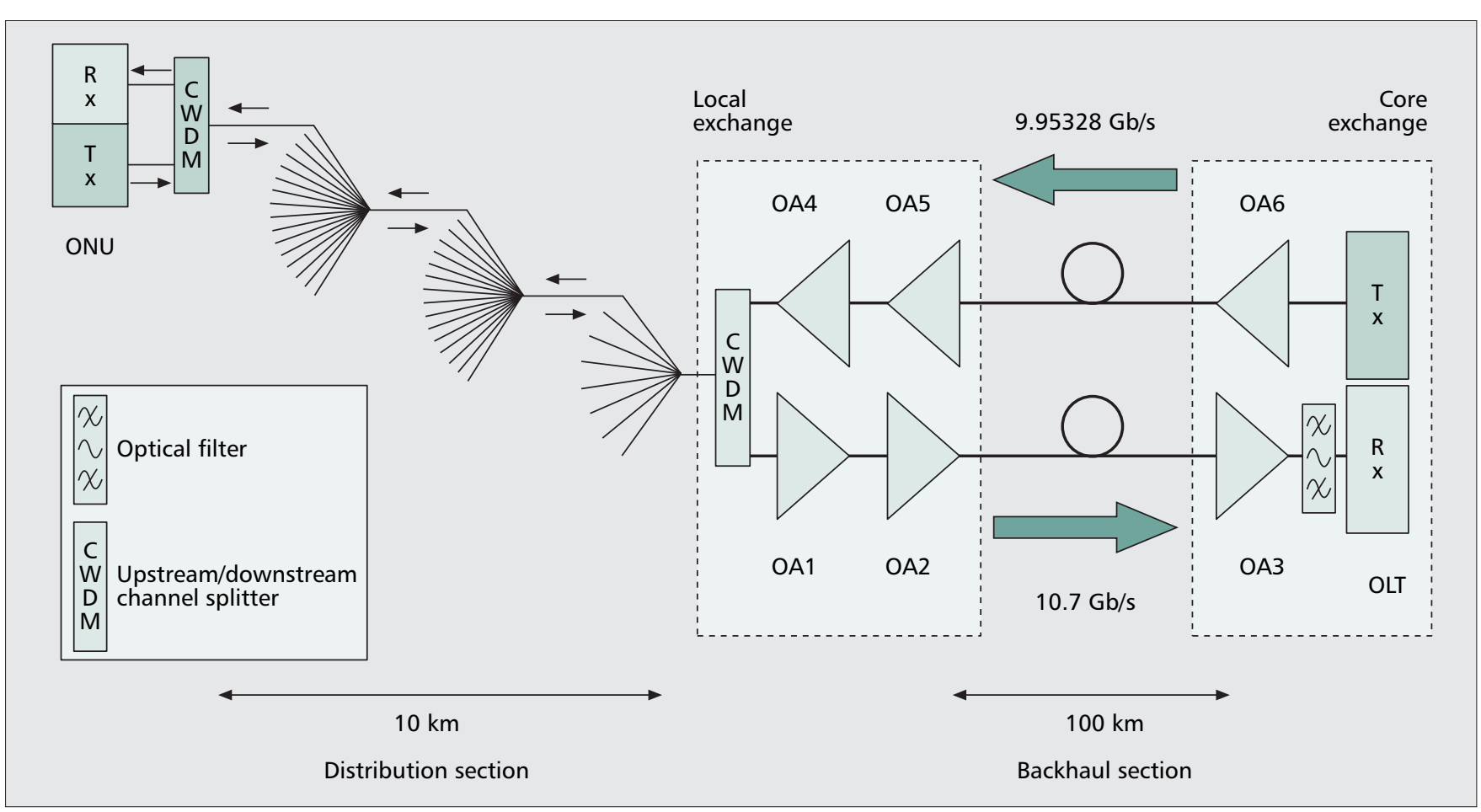

Figure 3. Long reach PON architecture [4].

exchange site into a single backhaul fiber using a DWDM multiplexer/demultiplexer. Both upstream and downstream use $100 \mathrm{Ghz}$ spaced channels that divide the C-band in half. Wavelengths from $1529 \mathrm{~nm}$ to $1541.6 \mathrm{~nm}$ are allocated to the downstream, and wavelengths from $1547.2 \mathrm{~nm}$ to $1560.1 \mathrm{~nm}$ are allocated to the upstream. This gives a total of 17 channels, enabling the network to support a total of 17 PONs with a $5 \mathrm{~nm}$ guard band between downstream and upstream wavelengths.

The local exchange site contains the DWDM sources for the centralized optical carrier generation along with the optical amplification stages. At the core exchange, after the backhaul fiber, the signal passes through a length of DCF to reduce the dispersion penalties. Upstream optical signals pass through an optical amplifier before being demultiplexed to each PON's OLT with an arrayed waveguide grating (AWG). Downstream signals are multiplexed with an AWG before being passed into the backhaul fiber.

This system offers $10 \mathrm{~Gb} / \mathrm{s}$ symmetrical upstream and downstream channels over $100 \mathrm{~km}$. The number of customers supported varies with the number of PONs that each backhaul section can support. With the current implementation, which supports 17 PONs and assumes each PON has a 256-way split, the total number of users was 4352 . Each user could access a minimum of $39 \mathrm{Mb} / \mathrm{s}$ (i.e., $10 \mathrm{~Gb} / \mathrm{s} / 256$ users).

\section{Long-Reach GPON}

A major obstacle for optical access networks was the cost associated with installing an optical transmitter and receiver in the ONU at the customer premises [2]. Standard PON reduces this burden by using lower cost uncooled transmitters in the ONU. However, this results in the transmission wavelength being temperature dependant with a possible drift of $20 \mathrm{~nm}$. As no component in a standard PON is wavelength critical, the performance was unaffected. The detrimental consequences of wavelength drift only become crucial when using narrow optical filters either for DWDM backhaul or ASE reduction. In these systems, more expensive cooled transmitters are used to ensure a stable wavelength.
DWDM GPON Extension - Extensive work was performed on the physical layer of long-reach optical access networks, but relatively little has been performed on the higher layer protocols that control the transmission over the physical layer. In recent work, the reach of a standard commercially available gigabit PON (GPON) was extended from $20 \mathrm{~km}$ to $135 \mathrm{~km}$ to assess the performance of the GPON protocols at this distance [6].

The experimental configuration, as shown in Fig. 5, consisted of a commercially available GPON with a 40-channel DWDM system, GPON ONUs, with standard upstream optics at $1310 \mathrm{~nm}$ and non-standard ONUs at $1510 \mathrm{~nm} \mathrm{[6].} \mathrm{The}$ upstream data rate was $1.25 \mathrm{~Gb} / \mathrm{s}$ that was transmitted across $10 \mathrm{~km}$ of standard single mode fiber and through a 64-way split to the bespoke transponder that aids in the conversion between the GPON and DWDM system. This unit contained a $1.25 \mathrm{~Gb} / \mathrm{s}$ burst mode receiver that converts the burst-mode GPON single-fiber network to a DWDM wavelength over a dual-fiber network. Then, the DWDM signal was transmitted over $125 \mathrm{~km}$ of fiber to the OLT; hence, the system has a total reach of $135 \mathrm{~km} \mathrm{[6].}$

The system successfully transported GPON data over the $135 \mathrm{~km}$ with a BER better than $10^{-10}$ [6]. This demonstrates that the standard GPON protocols can be easily extended to operate over long-reach access network distances when optical to electrical to optical (OEO) conversion is used.

\section{Wavelength Converting PON}

As we have seen, the reach of a GPON can be extended using OEO regeneration. However, all optical means are available. The wavelength converting (WC) PON uses optical wavelength conversion in the upstream to transfer the transmitted data from the ONU wavelength to a standard DWDM wavelength. In the wavelength conversion process, the network operator has complete control over the wavelength to which the original wavelength was converted. A number of PONs can be grouped together over the same backhaul fiber, with each PON being converted to a separate wavelength. This reduces the cost of the backhaul fiber on a per customer basis as the utilization and efficiency of the backhaul fiber is 


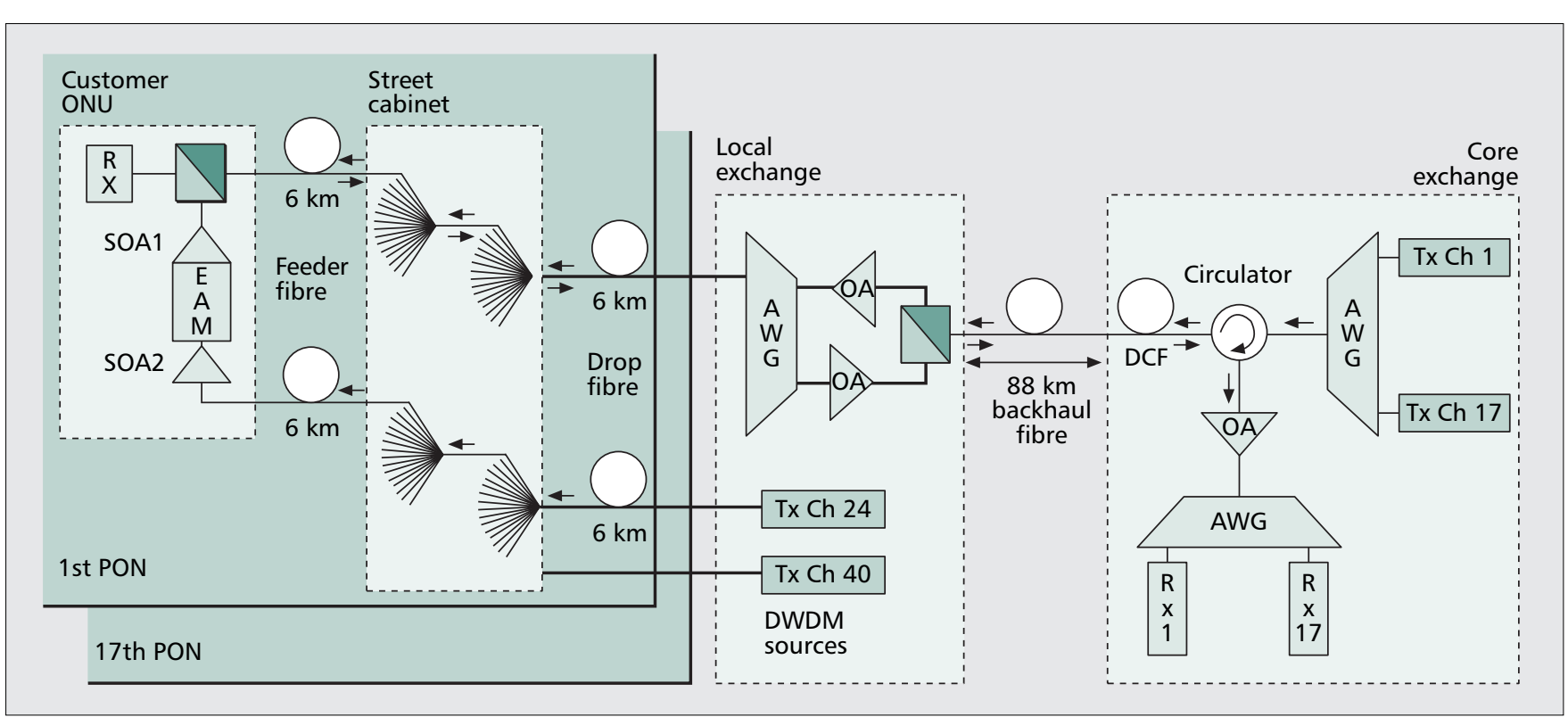

Figure 4. Hybrid DWDM-TDM PON architecture [5].

increased, enabling the cost to be spread over a large number of customers. Therefore, with the inclusion of a wavelength converter, a high split, long-reach optical access network that combines both access and metro networks can be created from standard PON architectures [7].

A demonstration of the upstream dual fiber architecture of the long-reach WC-PON is shown in Fig. 6. In the distribution section, an infrastructure that follows the GPON standard is represented by $20 \mathrm{~km}$ of standard single-mode fiber and a 64way split. The ONU transmitter consists of an uncooled transmitter with possible wavelength drift over a coarse wavelength division multiplexing channel (CWDM), that is, $18 \mathrm{~nm}$. In this implementation, a simple cross-gain modulation (XGM) wavelength converting technique was used due to its simplicity. The XGM technique places certain limits on the conversion bandwidth due to physical effects within the SOA. Therefore, the ONU wavelength was centered around 1550 $\mathrm{nm}$ and was free to drift within $\pm 9 \mathrm{~nm}$ of the center wavelength. A dual fiber backhaul was used to avoid any wavelength clashes between the upstream and downstream channels. The XGM wavelength converter consists of two stages: a SOA, where the XGM conversion was performed and a pre-amplifier stage. As the XGM wavelength conversion process relies on SOA gain saturation, an EDFA was used as a pre-amplifier stage to ensure there was sufficient optical signal power. Ideally, an SOA pre-amplifier would be used to aid integration. To improve the performance of the XGM wavelength conversion process, a CWDM filter $(18 \mathrm{~nm}, \lambda=1550 \mathrm{~nm})$ optical filter was used to reduce the SOA saturation through EDFA ASE. The wavelength that the signal was converted to (probe wavelength) was supplied by a continuous wave laser, injected into the SOA in the counter propagation direction. The signal on the converted wavelength was coupled out of the wavelength converter through a $2 \times 2$ coupler and multiplexed into a $100 \mathrm{~km}$ standard single mode fiber backhaul through an AWG. A second AWG was positioned immediately after the backhaul fiber to filter each chan- nel to the correct OLT. The AWG had 20 channels with 100 $\mathrm{GHz}$ bandwidth, enabling DWDM to be implemented. Therefore, it would be possible for the system to support 1280 users, over $120 \mathrm{~km}$. Each user would have access to a minimum symmetrical bandwidth of $38.8 \mathrm{Mb} / \mathrm{s}$ [7].

\section{PIEMAN}

Research is continuing into long-reach optical access as demonstrated by the Information Society Technologies (IST) sixth framework project (FP6), photonic integrated extended metro and access network (PIEMAN) [8]. The PIEMAN architecture is shown in Fig. 7. The network has a $100-\mathrm{km}$ reach with a 32 wavelength DWDM backhaul. Each $10 \mathrm{~Gb} / \mathrm{s}$ wavelength is uniquely allocated to a PON with a 512-way split, enabling the network to support $(32 \times 512) 16,384$ users with an average bandwidth of $\sim 20 \mathrm{Mb} / \mathrm{s}$. By using dynamic bandwidth allocation and $10 \mathrm{~Gb} / \mathrm{s}$ components in the ONU, it is possible for each user to burst at $10 \mathrm{~Gb} / \mathrm{s}$.

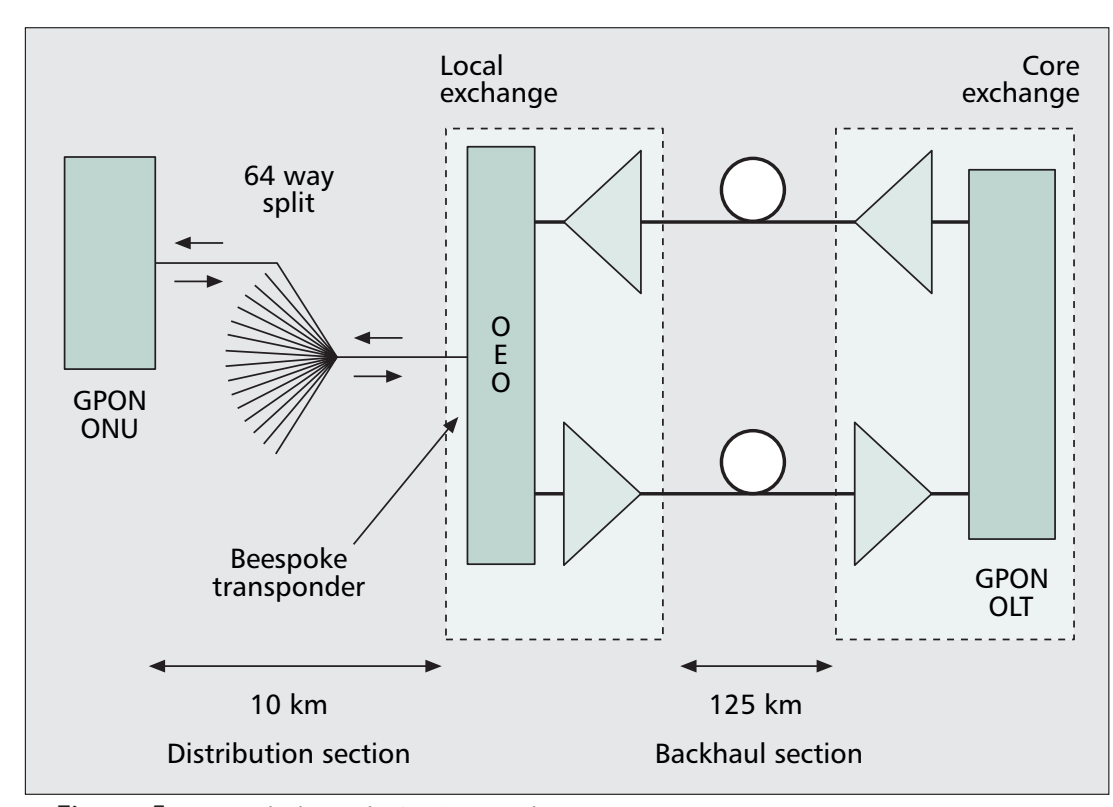

Figure 5. Extended reach GPON architecture. 


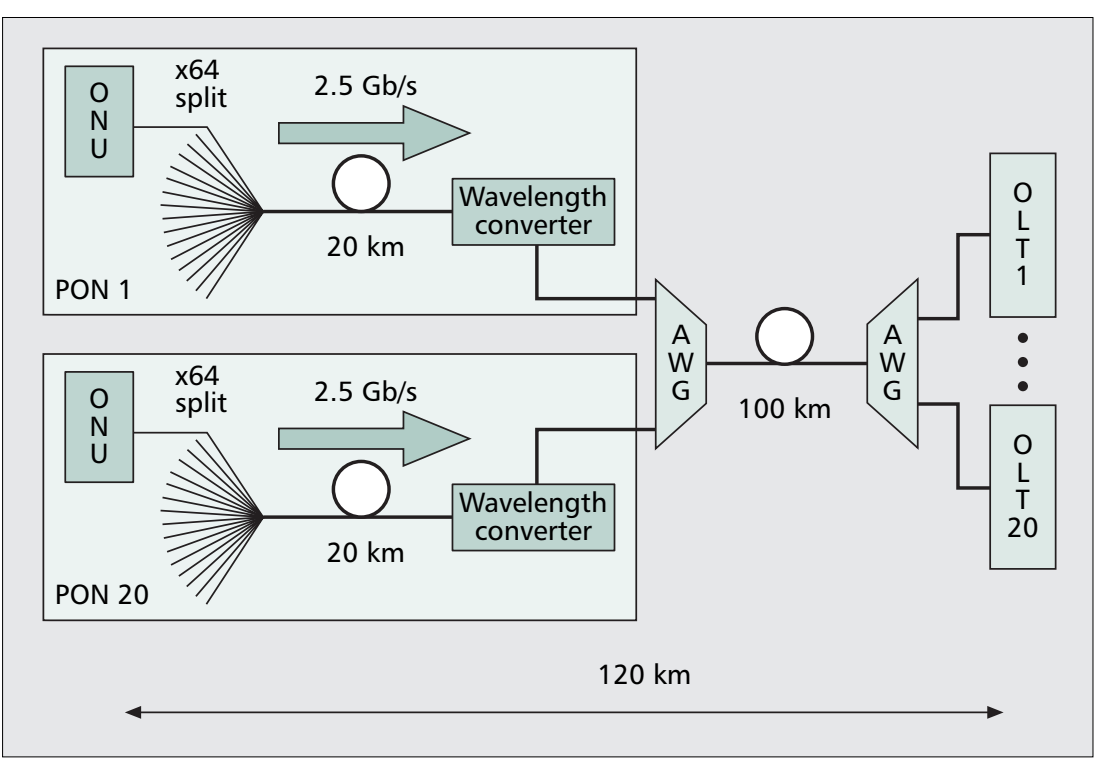

Figure 6. WC-PON architecture [7].

Colorless ONU will be provided for upstream transmission to avoid the requirement for each customer to buy an ONU with specific wavelength. Two components were identified as having the potential to provide a colorless ONU. The first is a tunable ONU that can select from 32 wavelengths with 50$\mathrm{GHz}$ spacing. To reduce the cost of the devices, a set and forget approach is taken for the tunable ONUs. Instead of tuning the ONU to a specific wavelength before transmitting, which requires the device to be wavelength agile, the wavelength of the device is set once when the ONU is added to the network, and no further changes to the ONU wavelength are made. A second approach under consideration is to use reflective SOAs. This approach is similar to the hybrid WDM/TDMA PON, where a wavelength is supplied to the ONU by the network. The reflective ONU then modulates, amplifies, and redirects the network supplied CW wavelength toward the OLT [8].

The burst mode nature of the TDMA traffic in the upstream path of PONs causes a number of problems. Standard EDFAs cannot be used due to the slow gain dynamics. That is, the gain of EDFA cannot change fast enough to ensure that the entire packet receives constant gain. The gain changes as the packet propagates through the EDFA causing the amplitude of the packet to be non-uniform. The selected solution uses an auxiliary wavelength that is adjusted relative to the transmitted upstream packet so that the optical power through the EDFA remains constant. Hence, the gain of the EDFA remains constant for the duration of the burst.

Upstream burst-mode traffic also means that a standard continuous mode receiver cannot be used. In current PONs, a DC coupled receiver is used, where the receiver must determine the correct threshold on a burst-by-burst basis due to

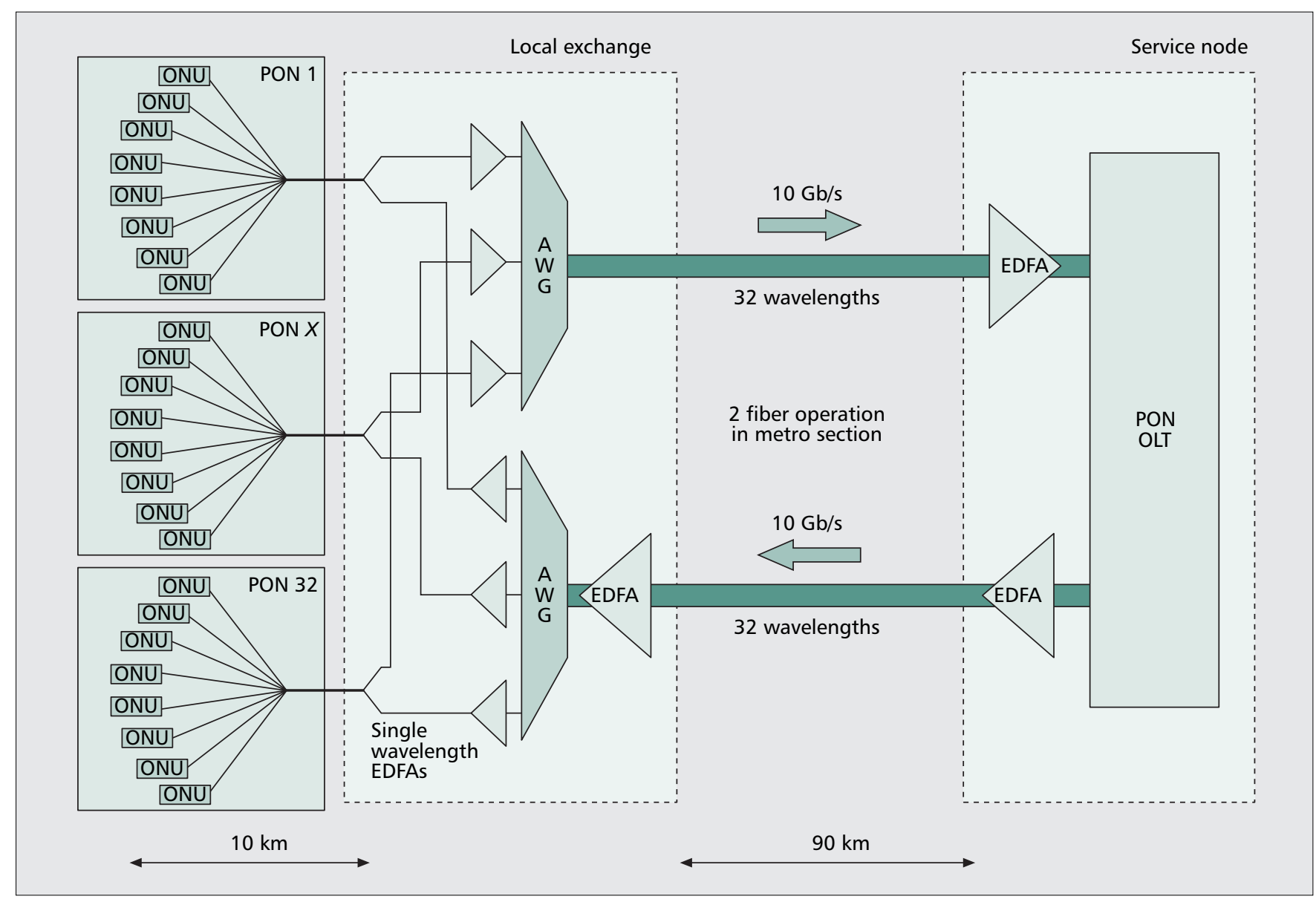

Figure 7. PIEMAN hybrid WDM/TDMA architecture [8]. 
path attenuation differences for different ONUs. Problems occur when scaling these techniques to $10 \mathrm{~Gb} / \mathrm{s}$. Hence, the PIEMAN project is developing a new $10 \mathrm{~Gb} / \mathrm{s}$ burst mode receiver that uses a multistage feed-forward architecture, reducing DC offsets through a long chain of electrical amplifiers [8].

\section{Conclusion}

A number of promising technologies have been developed that enable long-reach access to become a reality. SuperPON provided the foundation that proved the feasibility of providing FTTH along with network simplification through a combined access and metro network. Long-reach PON improved SuperPON by using modern components and techniques to provide $10 \mathrm{~Gb} / \mathrm{s}$ symmetrical bandwidth, but the choice of single-wavelength TDM/TDMA operation results in low efficiency in terms of bandwidth allocation and fiber utilization. The hybrid DWDM-TDM long-reach PON uses DWDM components to provide similar performance without the limitations of the long-reach PON. The PIEMAN project is advancing the WDM/TDM architecture and is researching technologies such as burst-mode receivers that will be vital when implementing optical access at $10 \mathrm{~Gb} / \mathrm{s}$. However, we believe that the most suitable systems for deployment of long-reach optical access will be the long-reach GPON and WC-PON. This is because these systems provide an intermediate step; enabling operators to deploy standard PONs now and then upgrade to long-reach in a simple manner.

\section{References}

[1] D. B. Payne and R. P. Davey, "The Future of Fiber Access Systems," BT Tech. J., vol. 20, 2002, pp. 104-14.

[2] D. W. Faulkner et al., "Optical Networks for Local Loop Applications," J. Lightwave Tech., vol. 7, 1989
[3] I. Van de Voorde et al., "The SuperPON Demonstrator: An Exploration of Possible Evolution Paths for Optical Access Networks," IEEE Commun. Mag. vol. 38,2000 , p. 74.

[4] D. P. Shea and J. E. Mitchell, "A $10 \mathrm{~Gb} / \mathrm{s} 1024-W a y$ Split 100-km Long Reach Optical Access Network," J. Lightwave Tech., vol. 25, no. 3, Mar. 2007.

[5] G. Talli and P. D. Townsend, "Feasibility Demonstrator of $100 \mathrm{~km}$ Reach DWDM SuperPON with Upstream Bit Rates of $2.5 \mathrm{~Gb} / \mathrm{s}$ and $10 \mathrm{~Gb} / \mathrm{s}$," OFC, Anaheim, CA, 2005.

[6] R. P. Davey et al., "DWDM Reach Extension of a GPON to $135 \mathrm{~km}$," OFC Tech. Digest, 2005.

[7] D. P. Shea and J. E. Mitchell, "Experimental Upstream Demonstration of a Long Reach Wavelength-Converting WDM-PON with DWDM Backhaul," OFC/NFOEC, Anaheim, CA, Mar. 25-29, 2007.

[8] G. Talli et al., "Integrated Metro and Access Network: PIEMAN," presented at 12 th European Conf. Networks and Opt. Commun., Kista, Sweden, June 2007, pp. 493-500.

\section{Biographies}

DARREN SHEA received his engineering doctorate (Eng.D.) degree in telecommunications from University College London (UCL), United Kingdom, in 2006. His thesis research, performed in conjunction with $\mathrm{BT}$, involved the design and construction of a $10 \mathrm{~Gb} / \mathrm{s}$ optical access network that connected 1024 customers directly to the core network over a distance of $100 \mathrm{~km}$. Prior to his doctorate studies, he received first class honors in electronic engineering from the University of Manchester. For the duration of his doctorate research, he worked as part of the Optical Networks group at BT, based at Adastral Park, Ipswich, United Kingdom. Currently, he is working as a research fellow at UCL. His research interests include optical access, specifically fiber to the home, and burst mode receiver and long haul transmission improvements using optical frequency chirping.

JOHN E. MITCHELL [S'97, M'00] (j.mitchell@ee.ucl.ac.uk) received his B.Eng. in electronic and electrical engineering and Ph.D. from UCL in 1996 and 2000 respectively. In 2000 he was appointed as a lecturer in the Department of Electronic and Electrical Engineering at UCL, becoming a senior lecturer in 2006. His research interests include optical access technologies (in particular long-reach and WDM-PONs) and the transport of millimeter wave radio signals over optical fiber for communications and noise relating to crosstalk in optical networks. He has published over 40 papers in the area of optical communications. He has presented invited talks and tutorials at a number of conferences, workshops, and meetings, both nationally and internationally, on the subject of radio-over-fiber networks and optical access. He is a member of the Institute of Engineering and Technology (IET) and the IEEE Lasers and Electro-Optics Society (IEEE-LEOS). 